\title{
An Analysis on the Solution of Curvature Constraint of $2+1$ Dimensional BF Model by Constructing a Projector $\mathbf{P}$
}

\author{
Diego C. M. Mendonça*; Olivier Piguet and Clisthenis P. Constantinidis \\ Universidade Federal do Espirito Santo, Brazil \\ E-mail: diegomendonca@gmail.com, opiguet@yahoo.com, \\ cpconstantinidis@gmail.com
}

\begin{abstract}
In this paper we analyze the structure of the physical Hilbert space of a $2+1$ dimensional BF model starting from a Hilbert space $\mathscr{H}_{0}$ of wave functionals which are gauge invariant, ie, the Gauss constraint $\mathscr{G}$ is considered to be already fulfilled. We see then that in $\mathscr{H}_{0}$ we have only the empty vector as a solution of the curvature constraint and we have to construct a larger space. This will be the dual $S^{\star}$ of a dense subspace $S \subset \mathscr{H}_{0}$. It is convenient to define an operator $P$, sometimes called "projector", which is a surjective mapping from the space $S$ to the physical Hilbert space $\mathscr{H}_{\text {fis }}$, subspace of the dual $S^{\star}$. If we can build the "projector" $P$ we have enough to define the inner product in space $\mathscr{H}_{\text {fis. }}$. In this work we see that the imposition of the curvature constraint $\mathscr{F}$ on the wave functional $|\Psi\rangle$ can be explicitly calculated with the aid of the Schwartz's theory of distributions. We use the technique known as group averaging in order to construct the projector and the physical Hilbert space, from a generalization to the present situation of the solution of a system of equations for Schwartz's distributions of one real variable.
\end{abstract}

4th International Conference on Fundamental Interactions

August 1-7, 2010

Viçosa, Brazil

\footnotetext{
*Speaker.

${ }^{\dagger}$ I thank FAPES and CAPES for supporting this work.
} 


\section{Introduction}

In this work we analyze the structure of the physical Hilbert space $\mathscr{H}_{\text {fis }}$ of a $2+1$ dimensional BF model $[1,2]$, ie, build the space in where one defines the wave functionals that obey the canonical constraints generators of symmetries $\mathscr{G}$ (Gauss constraint) e $\mathscr{F}$ (curvature constraint).

It is noteworthy that this work is a preliminary study where we use the results of Perez [3] to verify that our projector $P$ constructed via the Schwartz theory of distributions has the same form of the projector obtained by Perez [3, 4].

\section{The $2+1$ dimensional BF model}

The action of the BF model, invariant by diffeomorphisms and gauge transformations, is written as:

$$
S_{B F}=\operatorname{Tr} \int_{\mathscr{M}_{D}} B \wedge F(A)
$$

where $B$ is an 1-form and $F$ is the Yang-Mills curvature. The hamiltonian completely constrained can be obtained through a procedure of canonical quantization, where:

$$
H=\mathscr{G}(\lambda)+\mathscr{F}(\eta)
$$

makes clear that the dynamics of the theory is actually an arbitrary evolution given by the gauge transformations generated by the constraints. We write them explicitly:

$$
\begin{aligned}
\mathscr{G}(\lambda) & =\int d^{2} x \lambda^{I}(x) D_{a} \tilde{B}_{I}^{a}(x) \\
\mathscr{F}(\eta) & =\frac{1}{2} \int d^{2} x \eta_{I}(x) \varepsilon^{a b} F_{a b}^{I}(x)
\end{aligned}
$$

With the subsequent quantization of the theory we can only obtain physical states and describe observable operators if we can construct a physical Hilbert space $\mathscr{H}_{\text {fis }}$ from a kinematic space $\mathscr{H}_{\text {kin }}$, which involves solving the constraints to the quantum level.

We then consider the basis vectors $|s\rangle=|\Gamma, \vec{j}, \vec{v}\rangle \in \mathscr{H}_{0}$, where $\mathscr{H}_{0} \subset \mathscr{H}_{\text {kin }}$. Writing $\Psi_{s}=\langle A \mid s\rangle$ explicitly:

$$
\Psi_{s}[A]=\langle A \mid s\rangle \equiv\left(\bigotimes_{p} R^{\left(j_{p}\right)}\left(h_{\gamma_{p}}[A]\right)\right) \cdot\left(\bigotimes_{q} v_{i_{q}}\right)
$$

where $R^{\left(j_{p}\right)}$ are irreducible representations of the group of symmetries, which are functions of $h_{\gamma_{p}}[A]$, the holonomies of $A$ on a graph $\gamma_{p} . v_{i_{q}}$ are the intertwinners, quantities that keeps the vertices of the graph invariants. This basis, known as spin network[5, 6], is orthonormal and nonenumerable.

\section{The solution of the curvature constraint}

Let's take these $|\Psi\rangle \in \mathscr{H}_{0}$ to apply the second constraint of the theory, which is the zero spatial curvature constraint:

$$
\varepsilon^{a b} F_{a b}|\Psi\rangle=0
$$


As the only solution (3.1) in $\mathscr{H}_{0}$ is the "null" vector $|\phi\rangle$, corresponding to the empty graph[1], we must seek a larger space. This will be the dual $S^{\star}$ of a dense space $S$, which is subspace of the space $\mathscr{H}_{0}$. Hence, we introduce the Gelfand's triple:

$$
S \subset \mathscr{H}_{0} \subset S^{\star}
$$

where $S$ is the vector space of all finite linear combinations of vectors $|s\rangle$ of the spin network basis (2.5). As $S$ is dense in $\mathscr{H}_{0}$, this is the Cauchy completion of the space $S$. The dual $S^{\star}$ is composed of linear forms $\Phi$ in the vectors of $S$, with the notation of Schwartz:

$$
\Phi:|\Psi\rangle \in S \mapsto\langle\Phi, \Psi>\in \mathbb{C}
$$

It is convenient define an operator $P$, sometimes called "projector" which is a surjective mapping of space $S$ to the physical Hilbert space $\mathscr{H}_{\text {fis }}$, subspace of the dual $S^{\star}$ :

$$
P: S \rightarrow \mathscr{H}_{\text {fis }} \subset S^{\star}
$$

where $\mathscr{H}_{\text {fis }}$ is formed by vectors that obey the constraint (3.1). The definition of the projector $P$ is that every element $\Phi$ of $\mathscr{H}_{\text {fis }}$ is invariant under the gauge transformations $g$ generated by the constraint (3.1):

$$
U(g) \Phi=\Phi
$$

$U(g)$ is defined by duality, ie, $\forall \Psi \in S$ we have:

$$
<U(g) \Phi, \Psi>\equiv<\Phi, U^{\dagger}(g) \Psi>=\langle\Phi, \Psi\rangle
$$

where $U(g)$ is a unitary representation of the element $g$ of the gauge group. Assuming that we can build the "projector" $P$ with the above properties, we have enough to define the inner product in $\mathscr{H}_{\text {fis }}$ space:

$$
\left\langle\Phi_{1}, \Phi_{2}\right\rangle_{\text {fis }}=\left\langle P \Psi_{1}, \Psi_{2}>\right.
$$

where $\Phi_{1,2}=P \Psi_{1,2}$. It is shown [5] that if $\Phi_{1,2}=P \Psi_{1,2}^{\prime}$ (equivalence class), the physical inner product is independent of this difference between $\Psi$ :

$$
\left\langle P \Psi_{1}^{\prime}, \Psi_{2}^{\prime}\right\rangle=\left\langle P \Psi_{1}, \Psi_{2}\right\rangle
$$

the inner product of physical space does not depend on the corresponding $\Psi$ in space $S$.

\section{An example in the theory of functions of one variable}

This condition (3.1) on the wave functional $|\Psi\rangle$ can be calculated explicitly with the aid of the theory of Schwartz distributions. [7],[8] Consider an analogue of this condition in the theory of distributions of one real variable:

$$
(x-a) T(x)=0
$$


To this end, we write the Gelfand triple:

$$
S \subset L_{2} \subset S^{\star}
$$

where $S$ is the Schwartz space, dense in $L_{2}$, which is the space of rapidly decreasing functions that we call test functions and $S^{\star}$ is the dual, which is the space of continuous linear functionals of these test functions, the tempered distributions. So is the tempered distribution $T \in S^{\star}$ a linear functional $T: S \rightarrow \mathbb{C}$, than for a $\varphi \in S:$

$$
\varphi \mapsto<T, \varphi>\equiv \int d x T(x) \varphi(x)
$$

where the integral notation is symbolic. If $\Psi(x) \in L_{2}$ we can write the value of the distribution as:

$$
<\Psi, \varphi>=\int d x \Psi(x) \varphi(x)
$$

where $\int d x$ is the usual integral. The general solution of equation (4.1) is the distribution $T(x)=$ $c \delta(x-a)$, which can be verified directly by substitution. Let's examine the more general equation:

$$
f(x) T(x)=0
$$

The general solution of (4.5) for the case of zeros $x_{i}$ of order $n_{i}$ is:

$$
T(x)=\sum_{i} \sum_{l=0}^{n_{i}-1} c_{i l} \boldsymbol{\delta}^{(l)}\left(x-x_{i}\right)
$$

Note that both non degenerating and degenerate cases, the coefficients $c_{i}$ or $c_{i l}$ are completely arbitrary. A particular solution in non-degenerate case, considered here for its simplicity, is:

$$
T(x)=c \delta(f(x))=c \sum_{i} \frac{1}{\left|f^{\prime}\left(x_{i}\right)\right|} \delta\left(x-x_{i}\right)
$$

Note that this property creates a restriction for the coefficients $c_{i} l$ of equation (4.6) so that $\frac{c_{i}^{\prime}}{c_{j}^{\prime}}=$ $\left|\frac{f^{\prime}\left(x_{j}\right)}{f^{\prime}\left(x_{i}\right)}\right|$. We can now represent the delta function as the Fourier transform of a constant and so the particular solution (4.7) in the Fourier representation is:

$$
T(x)=c \delta(f(x))=\frac{1}{2 \pi} \int d k e^{i k f(x)}
$$

This result can be generalized to the degenerate case.

We saw how to solve the equation $f(x) T(x)=0$ for one real variable, and for our purpose of studying the condition $\mathscr{F}(A)|\Psi\rangle=0$ we have to take a system of equations for all real points $x$ where the connection $A$ is defined, through a procedure equivalent to a regularization of space.

\section{The construction of the projector $P$}

We got back to the projector $P$ (3.4). There is a technique known as group averaging [9],[3], that allows the definition of this projector $P$ by a regularization in order to generalize the results to the theory of distributions of a system of equations of one real variable. 


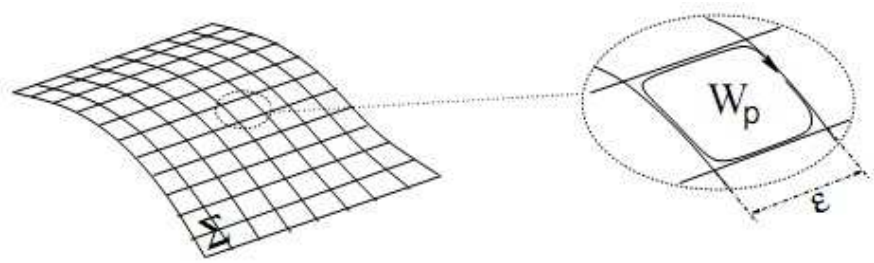

Figure 1: Figure 1: Cellular decomposition of the manifold $\Sigma$, with a network element of width $\varepsilon$ and a plaquette $p$ where we have an infinitesimal holonomy $W_{p}[A]$.

Be the group $G$ of all gauge transformations. Before treating the general case, we take a finite group $G$ of $N$ elements, and try to solve the condition of invariance under $G$ :

$$
U(g)|\Psi\rangle=|\Psi\rangle \quad \forall U(g) \in G
$$

The solution of these equations is the average over the group:

$$
|\Psi\rangle=\frac{1}{N} \sum_{U \in G} U(g)|\Psi\rangle \equiv P|\Psi\rangle
$$

so $P$ is nothing more than proportional to the sum of all transformations of the group $G$. In the case of $G$ be the group of unitary representations $U(g)$ of the gauge transformations from $\mathscr{F}$ with parameters $N_{I}(x)$ :

$$
U[N]=e^{i \int d^{2} x N_{I}(x) F^{I}(x)}=e^{i \mathscr{F}[N]}
$$

where $\mathscr{F}$ is the generator (2.4) of these gauge transformations, ie, the constraint (3.1). The projector $P$ is the sum over all the possible $\mathscr{F}$ gauge transformations, which generates a functional integration in $N$. Formally:

$$
P=\int \mathscr{D} \mathscr{N} e^{i \int d^{2} x N_{I} F^{I}}=\delta\left(F^{I}(A)\right)
$$

Note the similarity of this result with equation (4.8) for one real variable that we found in a wholly different context. This shows that taking a proper regularization, we can use the example of a Schwartz's distribution to build our projector $P$ (see Fig 1).

To define better the expression (5.4) we write first the constraint $\mathscr{F}(2.4)$ at the classical level, as the limit of a Riemann sum:

$$
\mathscr{F}[N]=\int_{\Sigma} \operatorname{Tr}[N F(A)]=\lim _{\varepsilon \rightarrow 0} \sum_{p} \varepsilon^{2} \operatorname{Tr}\left[N_{p} F_{p}\right]
$$

where $N_{p}$ e $F_{p}$ are the values of the parameter $N$ and the curvature $F$ in an interior point of the plaquette $p$ (Fig. 1). The holonomy $W_{p}$ defined around the edges of the plaquette $p$ can be calculated:

$$
W_{p}[A]=1+\varepsilon^{2} F_{p}(A)+\mathscr{O}\left(\varepsilon^{3}\right)
$$


The equations (5.5) and (5.6) imply:

$$
\mathscr{F}[N]=\lim _{\varepsilon \rightarrow 0} \sum_{p} \operatorname{Tr}\left[N_{p} W_{p}\right]
$$

With this, we will be able to express the regulated constraint around holonomies whose action on the vectors of $\mathscr{H}_{0}$ is well defined. With this result, we can write the inner product of spin network states (3.7) projected into physical space, which will be of the form:

$$
\left\langle s^{\prime} P, s\right\rangle_{\text {fis }}=\lim _{\varepsilon \rightarrow 0} \prod_{p}^{n_{p}(\varepsilon)} \sum_{j_{p}}\left(2 j_{p}+1\right)\left\langle s^{\prime} \operatorname{Tr}\left[R^{\left(j_{p}\right)}\left(W_{p}\right)\right], s\right\rangle
$$

where $j_{p}$ is the associated spin to $p$-th plaquette and $\operatorname{Tr} R^{\left(j_{p}\right)}\left(W_{p}\right)$ is the character of the unitary irreducible representation of spin $j_{p}$ for $S U(2)$. There is also in [9],[4] that the limit $\varepsilon \rightarrow 0$ exists.

This regulated expression is a linear form on $\mathscr{H}_{\text {fis }}$, and when these matrix elements are computed on an invariant spin network basis they can be expressed as spin foams, as seen in [9],[3].

\section{References}

[1] A. Ashtekar, J. Lewandowski, Background Independent Quantum Gravity: A Status Report, arxiv.org September 2004 [arXiv: gr-qc/ $0404018 v 2$ ].

[2] S. Carlip, Quantum Gravity in 2+1 dimensions, Cambridge University Press, Cambridge 1998.

[3] A. Perez, Spin foam models for quantum gravity, Class. Quant. Grav., 20:R43 2003.

[4] K. Noui, A. Perez, Three dimensional loop quantum gravity: Physical scalar product and spin foam models, arxiv.org 2004 [arXiv: gr-qc/ 0402110 ].

[5] C. Rovelli, Quantum Gravity, Cambridge University Press, Cambridge 2004.

[6] T. Thiemann, Modern Canonical Quantum General Relativity, Cambridge University Press, Cambridge 2007.

[7] W. Rudin, Functional Analysis, McGraw-Hill Education, India 2006.

[8] I. F. Wilde, Distribution Theory (Generalized Functions) Lecture Notes, Department of Mathematics King's College, London.

[9] A. Perez, Introduction to Loop Quantum Gravity and Spin Foams, Proceedings of the II International Conference on Fundamental Interactions, Pedra Azul, Brazil June 2004. 